IOS Press

\title{
Golgi phosphoprotein 3 (GOLPH3) promotes endometrial carcinoma cell invasion and migration by regulating the epithelial- mesenchymal transition
}

\author{
Yu Wen ${ }^{\mathrm{a}, \mathrm{b}}$, Xiaoqing Tan ${ }^{\mathrm{b}}$, Xia Wu ${ }^{\mathrm{b}}$, Qin $\mathrm{Wu}^{\mathrm{b}}$, Yan Qin ${ }^{\mathrm{b}}$, Miao Liang ${ }^{\mathrm{b}}$, Guangqin $\operatorname{Ran}^{\mathrm{b}}$, \\ Huiying $\mathrm{Gu}^{\mathrm{c}, *}$ and Rongkai Xie ${ }^{\mathrm{a}, *}$ \\ ${ }^{a}$ Department of Obstetrics and Gynecology, The Second Affiliated Hospital, Army Medical University, Chongqing \\ 400037, China \\ ${ }^{\mathrm{b}}$ Department of Obstetrics and Gynecology, Chongqing General Hospital, University of Chinese Academy of \\ Science, Chongqing 40014, China \\ ${ }^{\mathrm{c}}$ Department of Hepatobiliary Surgery, The Second Affiliated Hospital, Army Medical University, Chongqing \\ 400037, China
}

\begin{abstract}
.
BACKGROUND: Golgi phosphoprotein 3 (GOLPH3) is a novel oncogene overexpressed in several human cancers, but specific contributions to endometrial carcinoma (EC) have not been examined. The aims of this study were to evaluate the GOLPH3 expression in $\mathrm{EC}$ and investigate its functions in EC cell proliferation, migration, and survival.

METHODS: The expression levels of GOLPH3 in EC patient samples and EC cell lines (HEC-1A, KLE, RL95-2, and Ishikawa) were examined using qRT-PCR, western blotting and immunohistochemistry. Further, EC cell lines with either ectopic GOLPH3 overexpression or knockdown were established, and the effects on proliferation, apoptosis, invasion, and migration were investigated in vitro using cell viability and transwell assays and in mice following cell injection.

RESULTS: Compared to adjacent non-cancerous tissues, expression of GOLPH3 was significantly upregulated in EC tissues $(P<0.05)$, and the expression level of GOPLPH3 was related to the grade of the tumor $(P<0.05)$. The expression of GOLPH3 was also higher in all four EC cell lines than endometrial stromal cells (ESCs) $(P<0.05)$. Moreover, GOLPH3 expression was greater in EC cell lines with high invasive capacity than in non-invasive EC cells $(P<0.05)$. Knockdown of GOLPH3 inhibited EC cell proliferation and increased cell apoptosis in vitro. Further, knockdown of GOLPH3 also inhibited EC cell invasion and migration in vitro and in vivo by regulating the epithelial-mesenchymal transition (EMT). Conversely, GOLPH3 overexpression promoted proliferation and migration.

CONCLUSIONS: The present study provides evidence that GOLPH3 promotes EMT and metastasis of EC cells and predicts the risk of EC progression, highlighting its potential as a therapeutic target for this malignancy.
\end{abstract}

Keywords: GOLPH3, endometrial carcinoma, proliferation, invasion, migration, EMT

* Corresponding authors: Huiying Gu, Department of Hepatobiliary Surgery, The Second Affiliated Hospital, Army Medical University, Chongqing 400037, China. Tel.: +86 23 68763326; Fax: +86 23 68763326; E-mail: jainvephraly@163.com; Rongkai Xie, Department of Obstetrics and Gynecology, the Second Affiliated Hospital, Army Medical University, Chongqing 400037, China. Tel.: +86 13883818192; Fax: +86 23 68774606; E-mail: xqtrmyy@163.com.
Abbreviations
GOLPH3 Golgi phosphoprotein 3
EC endometrial carcinoma
ESC endometrial stromal cell
NAT normal adjacent tissue
EMT epithelial-mesenchymal transition
MET mesenchymal-epithelial transition

ISSN 1574-0153/19/\$35.00 (C) 2019 - IOS Press and the authors. All rights reserved 


$\begin{array}{ll}\text { mTOR } & \text { mechanistic target of rapamycin } \\ \text { NF- } \kappa \text { B } & \text { nuclear factor } \kappa \text { appa B } \\ \text { CCK-8 } & \text { Cell Counting Kit-8 } \\ \text { RPMI-1640 } & \text { Roswell Park Memorial Institute } \\ \text { ATCC } & \text { American Type Culture Collection } \\ \text { DMEM } & \begin{array}{l}\text { Dulbecco's modified Eagle's } \\ \text { medium }\end{array} \\ \text { FBS } & \text { fetal bovine serum } \\ \text { RIPA } & \text { radio-immunoprecipitation assay } \\ \text { PVDF } & \text { polyvinylidene difluoride } \\ \text { shRNA } & \begin{array}{l}\text { short hairpin RNA } \\ \text { standard deviation }\end{array} \\ \text { SD } & \begin{array}{l}\text { reverse transcription polymerase } \\ \text { RT-PCR }\end{array} \\ \text { IHC } & \begin{array}{l}\text { immun reaction } \\ \text { SDistochemistry }\end{array} \\ & \text { lamide dodecyl sulfate polyacry- } \\ & \end{array}$

\section{Introduction}

Endometrial carcinoma (EC) is the most common malignancy of the female reproductive tract in developed countries [1]. In recent years, EC incidence has increased worldwide, posing a further threat to female health. In many cases the prognosis is satisfying, but $22 \%$ of affected women die from the disease. While most cases are diagnosed in the early stages, nearly a third present with regional or distant metastasis. Moreover, despite recent therapeutic advances, metastasizing EC still has a low 5-year survival rate and a high rate of recurrence [2]. The elucidation of molecular pathways underlying tumorigenesis and progression of EC is thus critical for the identification of prognostic factors and the development of diagnostic tests and targeted molecular therapies $[3,4]$.

Golgi phosphoprotein 3 (GOLPH3) is a highly conserved 34-kDa trans-Golgi matrix membrane protein involved in receptor recycling, glycosylation, and protein trafficking [5-7]. Recently, GOLPH3 expression was found to correlate with clinical stage, growth, survival, and metastasis of several cancers, including small cell lung cancer [8], breast cancer [7,9], ovarian epithelial cancer [10], oral tongue cancer [11], liver cancer [12], rectal cancer [13], prostate cancer [14], bladder cancer [15], esophageal squamous cell carcinoma [16], gastric cancer [17,18], and kidney cancer [19]. It is suggested that GOLPH3 functions as an oncogene in tumorigenesis and migration by activating the mechanistic target of rapamycin (mTOR), Wnt, and nuclear factor $\kappa$-B (NF- $\kappa \mathrm{B})$ signaling path- ways [20-22]. However, GOLPH3 functions in the development of EC have not been reported.

The present study examined the expression of GOLPH3 in tissue samples of EC patients and EC cell lines. We then investigated the possible roles of GOLPH3 in EC cell proliferation, apoptosis, invasion, migration, and epithelial-mesenchymal transition (EMT) by manipulating its expression in EC cell lines in vitro and in animal models.

\section{Materials and methods}

\subsection{Design of the study}

The expression level of GOLPH3 in EC tissues and EC cell lines were detected by qRT-PCR, western blot and immunohistochemistry to investigate whether GOLPH3 involve in EC development. And In order to analyze the possible roles of GOLPH3 in EC cells, GOLPH3 shRNA and pcDNA3.1 (+)-GOLPH3 plasmid were constructed and transfected into KLE EC cell line and Ishikawa cell line, respectively, the effects of GOLPH3 on EC cell proliferation (by using CCK8 cell viability assay), migration and invasion (by using transwell assays and Xenograft Tumor model assay) and apoptosis (by using Annexin V FITC apoptosis detection assays) were examined. The levels of EMT-related genes in overexpression and knockdown EC cells were detected by qRT-PCR and western blot to elucidate the mechanism of GOLPH3 involvement in cell migration and invasion.

\subsection{Patient information and tissue specimens}

Thirty EC tissue samples and adjacent non-cancerous tissues were randomly collected from EC patients who underwent curative resection from 1/2014 to $12 / 2015$ at the Second Affiliated Hospital of Army Medical University. And endometrial tissue specimen employed in this study was obtained from an endometriotic patient who had no history of endometrial hyperplasia or neoplasia, and had not received any antiinflammatory or hormonal medications during a period of at least three months before surgery. Study protocols were reviewed and approved by the Ethics Committee of the Second Affiliated Hospital of Army Medical University and conducted according to the principles expressed in the Declaration of Helsinki. Informed written consent was provided by both patients and families before resection surgery. 


\subsection{Tissue chip}

The endometrial carcinoma tissue chip was purchased from Shanghai Outdo Biotech Co. Ltd., with the CGT number HUteA060CS01 and the lot number XT15-033. The chip contained 34 endometrial carcinoma tissues and 26 normal endometrial tissues derived from 34 cases, which were fixed in 60 pores.

\subsection{Chemicals and antibodies}

Lipofectamine transfection (Cat. No. L3000-015) and TRIzol (Cat. No. 15596026) reagents were purchased from Life Technologies (Grand Island, NY, USA). Anti-GOLPH3 antibody (Cat. No. ab82377, 1:100) and an Annexin V FITC apoptosis detection kit (Cat. No. ab14085) were purchased from Abcam (Cambridge, MA, USA). Peroxidase-labeled antirabbit IgG (H+L) antibody (Cat. No. A0208) was purchased from Beyotime (Shanghai, China). Antibodies against N-cadherin (Cat. No. 4061, 1:500), vimentin (Cat. No. 3932, 1:500), E-cadherin (Cat. No. 3195, 1:500), $\alpha$-catenin (Cat. No. 2131, 1:500), and GAPDH (Cat. No. 3683, 1:800) were purchased from Cell Signaling Technology (Danvers, MA, USA). A CCK-8 kit (Cat. No. CK04) was purchased from DOJINDO (Minato-ku, TKY, Japan). Transwell plates with $8-\mu \mathrm{m}$ pore filters (Cat. No. 3374) were purchased from Corning (Corning, NY, USA). Matrigel (Cat. No. 354234) was purchased from BD Biosciences (Franklin Lakes, NJ, USA). Unless otherwise noted, all other chemicals were purchased from Sigma (St. Louis, MO, USA).

\subsection{Cell lines and cell culture}

The human EC cell lines HEC-1A, KLE, RL952, and Ishikawa were purchased from American Type Culture Collection (ATCC, Rockville, MD, USA). Endometrial stromal cells (ESC) were obtained from the endometrial tissue specimen, Which taken from an endometriotic patient and separated as described by Hou et al. [23]. Cells were cultured at $37^{\circ} \mathrm{C}$ in Dulbecco's modified Eagle's medium (DMEM, Cat. No. 11965092, Gibco) or Roswell Park Memorial Institute-1640 (RPMI-1640, Cat. No. 11875-093, Gibco) supplemented with $10 \%$ fetal bovine serum (FBS, Cat. No. SV30087, HyClone, Logan, Utah, USA) under a humidified atmosphere containing $5 \% \mathrm{CO}_{2}$.

\subsection{Vectors, retroviral infection, and transfection}

For ectopic overexpression, a GOLPH3 expression construct was generated by subcloning PCRamplified full-length human GOLPH3 cDNA into the pcDNA3.1 (+) plasmid, the empty vector was used as a negative control. For GOLPH3 knockdown, human GOLPH3-targeting short hairpin RNA (shRNA) oligonucleotide sequences were cloned into the pSuper-retro-puro vector. The shRNA sequences were RNAi-1 5'-GCAGCGCCTCATCAAGAAAGT3' and RNAi-2 5'-GCATGTTAAGGAAACTCAGCC3', a scrambled shRNA ( ative control, the sequence of the scrambled shRNA was 5'-TTCTCCGAACGTGTCACGTTT-3' (synthesized by Invitrogen). Transfection of plasmids was performed using Lipofectamine 3000 reagent (Invitrogen, Carlsbad, CA, USA) according to the manufacturer's instructions, and the transfection efficiency was evaluated by $q R T-P C R$ and Western blotting.

\section{7. $R T-P C R$ and $q R T-P C R$}

Cells were dissociated with $0.25 \%$ trypsin (Invitrogen) and collected for reverse transcription polymerase chained reaction (RT-PCR). Total RNA was isolated using TRIzol reagent (Invitrogen) and cDNA was synthesized using SuperScript II reverse transcriptase. Primers were synthesized by Invitrogen with the assistance of Primer Premier 5.0 (PREMIER Biosoft International, Palo Alto, CA, USA). Sequences used in this study are listed in Table 1. GAPDH was used as the internal control. Quantitative (q)RT-PCR and data collection were performed using an ABI PRISM 7900HT sequence detection system and expression levels analyzed using the comparative threshold cycle $\left(2^{-\Delta \Delta C T}\right)$ method.

\subsection{Western blotting}

Cells were washed and treated with radio-immunoprecipitation assay (RIPA) lysis buffer. Cell lysates were centrifuged and the supernatant proteins mixed with loading buffer and boiled for $10 \mathrm{~min}$. Protein samples were separated by sodium dodecyl sulfate polyacrylamide gel electrophoresis (SDS-PAGE) and blotted onto polyvinylidene difluoride (PVDF) membranes. After blocking in non-fat milk for $1 \mathrm{~h}$ at room temperature, the membranes were incubated with primary antibodies for $12 \mathrm{~h}$ at $4{ }^{\circ} \mathrm{C}$ and peroxidaseconjugate secondary antibody for $1 \mathrm{~h}$ at room tempera- 
Table 1

Primer sequences used for qRT-PCR

\begin{tabular}{ll}
\hline Primer & Sequence (5'-3') \\
\hline GOLPH3-Forward & GGG CGA CTC CAA GGA AAC \\
GOLPH3-Reverse & CAG CCA CGT AAT CCA GAT GAT \\
Ki67-Forward & CCA CGA GAC GCC TGG TTA CTA T \\
Ki67-Reverse & GCC TCC TGC TCA TGG ATT TCA A \\
N-cadherin-Forward & CCG GTT TCA TTT GAG GGC ACA TGC \\
N-cadherin-Reverse & GCC GTG GCT GTG TTT GAA AGG C \\
Vimentin-Forward & AAC TTA GGG GCG CTC TTG TC \\
Vimentin-Reverse & GGT GGA CGT AGT CAC GTA GC \\
E-cadherin-Forward & TGG GCT GGA CCG AGA GAG TTT C \\
E-cadherin-Reverse & ATC CAG CAC ATC CAC GGT GAC G \\
$\alpha$-catenin-Forward & TCA TTG TGG ACC CCT TGA GC \\
$\alpha$-catenin-Reverse & TTA CGT CCA GCA TTG CCC AT \\
GAPDH-Forward & GCT GGC GCT GAG TAC GTC GT \\
GAPDH-Reverse & ACG TTG GCA GTG GGG ACA CG \\
\hline
\end{tabular}

ture. The bands were visualized by enhanced chemiluminescence (Amersham Biosciences, Little Chalfont, UK) and protein expression estimated by densitometry using Image $\mathrm{J}$.

\subsection{Immunohistochemistry}

For immunohistochemistry (IHC), the paraffin tissue chip was dehydrated in PBS and underwent antigen retrieval in sodium citrate buffer $(10 \mathrm{mM}, 0.05 \%$ Tween 20, pH 6.0). Then the tissue chip was blocked in $10 \%$ normal serum with $1 \%$ BSA in TBS for $2 \mathrm{hr}$ at room temperature. The chip was incubated with AntiGOLPH3 antibody for $12 \mathrm{~h}$ at $4{ }^{\circ} \mathrm{C}$ and peroxidaselabeled anti-rabbit $\mathrm{IgG}(\mathrm{H}+\mathrm{L})$ antibody for $1 \mathrm{~h}$ at room temperature. Then incubated with DAB chromogen for $1 \sim 10$ minutes until it got the appropriate dyeing intensity, and counterstained with hematoxylin.

\subsection{0. $C C K-8$ cell viability assay}

Cells were seeded onto 96 -well plates at $3 \times 10^{5}$ cells/well in $100 \mu \mathrm{L}$ culture medium containing $10 \%$ FBS and cultured for 24, 48, 72, and $96 \mathrm{~h}$. At the indicated times, CCK-8 solution $(10 \mu \mathrm{L})$ was added to each well and incubated for $1-4$ hours at $37^{\circ} \mathrm{C}$ under $5 \% \mathrm{CO}_{2}$. The plate was mixed gently on an orbital shaker for 1 minute to ensure homogeneous distribution of color, and then absorbance was measured at $450 \mathrm{~nm}$ using an ultraviolet spectrometer (Beckman Coulter, Brea, CA, USA).

\subsection{Apoptosis assay}

Cells were seeded at $1 \times 10^{6}$ per $10-\mathrm{cm}$ plate and incubated for $24 \mathrm{~h}$. Cell morphology was assessed by phase-contrast microscopy. Cells were then harvested using trypsin-EDTA, washed twice with PBS, and resuspended in binding buffer at $10^{6}$ cells $/ \mathrm{ml}$. Annexin $\mathrm{V}$ and propidium iodide were added (each at $5 \mu \mathrm{l} / 10^{5}$ cells) and the suspension incubated for $15 \mathrm{~min}$ at room temperature in the dark. The proportion of apoptotic cells was analyzed using an EPICS XL flow cytometer (Beckman Coulter).

\subsection{Invasion and migration assays}

Invasion assays were performed using Matrigelcoated transwell inserts (Costar, Manassas, VA, USA) containing polycarbonate filters with $8-\mu \mathrm{m}$ pores. According to the manufacturer's instructions, the inserts were coated with $50 \mu \mathrm{l}$ of $1 \mathrm{mg} / \mathrm{ml}$ Matrigel matrix. Cells were seeded on the upper chamber surface at $2 \times 10^{5}$ cells per $200 \mu \mathrm{l}$ of serum-free medium, and $600 \mu \mathrm{l}$ of medium with $10 \%$ FBS was added to the lower chamber. After 24 hours' incubation, cells that migrated to the lower surface of the membrane were fixed in $4 \%$ paraformaldehyde and stained by $0.1 \%$ crystal violet solution. Migrated cells in five random fields were counted on each membrane at $\times 100 \mathrm{Mag}-$ nification, cells were counted under an Olympus fluorescence microscope (Tokyo, Japan). Migration assays were conducted in a similar manner except that the transwell inserts were not coated with Matrigel.

\subsection{In vivo tumor metastasis}

Female Balb/c nude mice (total number $=14$, aged 4-5 weeks, weight 18-20 g) were purchased from the Laboratory Animal Center of third medical university and were humanely housed in barrier facilities on a $12 \mathrm{~h}$ light/dark cycle, Water and food were freely avail- 
able at all times.. Cells lines prepared as described were resuspended in PBS at $1 \times 10^{7}$ cells $/ \mathrm{ml}$. A suspension $(0.1 \mathrm{ml})$ of control or GOLPH3-knockdown cells was injected into the tail vein of nude mice $(n=$ 7/group). Animals were sacrificed 30 days after inoculation by the method of cervical dislocation, and tumor volume calculated as (length $\times$ width $\left.^{2}\right) / 2$, Length and width measuredments were obtained with calipers. All experimental procedures were approved by the Institutional Animal Care and Use Committee of Third Medical University.

\subsection{Statistical analysis}

All measurement data were presented as means \pm standard deviation (SD), and were analyzed using Graph Pad Prism software (GraphPad Software Inc, version 7.0). Student's two-tailed $t$-test was employed for comparisons between two groups, one-way ANOVA was performed for multiple comparisons, followed by Bonferroni's post hoc test. All enumeration data were expressed in number, the correlation of GOLPH3 and the clinic-pathological factors was analyzed using Mann-Whitney analysis or Chi-square test. Results were considered significant at $P<0.05$ and are indicated in the figures by asterisks $\left({ }^{*} p<0.05\right.$; $\left.{ }^{* *} p<0.01\right)$.

\section{Results}

\subsection{GOLPH3 is highly expressed in endometrial carcinoma (EC) and correlated with distant metastasis}

To investigate GOLPH3 involvement in EC, mRNA and protein levels in four EC cell lines were determined by qRT-PCR and western blot, respectively, ki67 was detected as positive control. Results revealed significantly higher expression levels in all lines compared to non-malignant endometrial stromal cells (ESCs) (Fig. 1A and B). We further analyzed the relationship between GOLPH3 expression and metastasis capacity in EC cell lines by transwell migration, and found higher expression in the invasive EC cell lines KLE and HEC-1A than the non-invasive EC cell lines RL95-2 and Ishikawa (Fig. 1A and B). Analysis of $30 \mathrm{EC}$ and matched normal adjacent tissue (NAT) samples by qRT-PCR and 6 EC/NAT samples by western blot revealed significantly higher GOLPH3 mRNA and protein expression in tumor tissue (Fig. 1C and D).
Table 2

The Correlation between GOLPH3 protein and clinic-pathological factors in endometrial carcinoma tissues $(n=34)$

\begin{tabular}{lrcc}
\hline \multirow{2}{*}{ Factor } & \multicolumn{2}{c}{ GOLPH3 } & \multirow{2}{*}{$\begin{array}{c}P \\
\text { value }\end{array}$} \\
\cline { 2 - 3 } & Positive & Negative & $1.000^{\mathrm{a}}$ \\
\hline Age (years) & 8 & 3 & \\
$\quad \leqslant 50$ & 17 & 6 & \\
$>50$ & & & $0.037^{\mathrm{b}, *}$ \\
Grade & 3 & 0 & \\
I & 17 & 4 & \\
II & 5 & 5 & \\
III & & & $0.347^{\mathrm{b}}$ \\
Stage & 14 & 4 & \\
I & 6 & 2 & \\
II & 5 & 1 & \\
III & 0 & 2 & \\
IV & & & $0.435^{\mathrm{a}}$ \\
Lymph node metastasis & 9 & 5 & \\
Yes & 16 & 4 & \\
No & & & \\
\hline
\end{tabular}

a : analyzed by Chi-square test; ${ }^{\mathrm{b}}$ : analyzed by Mann-Whitney analysis; ${ }^{*} P<0.05$.

Then we carried out IHC to determine the GOLPH3 localization in endometrial carcinoma and the relationship between GOLPH3 expression level and clinical factors of EC. The staining showed that GOLPH3 were overexpressed in the cytoplasm of carcinoma tissues (Fig. 1E). Further, the expression of GOLPH3 was related to the endometrial carcinoma grade. However, no correlation was found between GOLPH3 expression and other clinical factors, such as age, stage and lymph node metastasis (Table 2).

\subsection{Suppression of GOLPH3 expression reduces EC cell proliferation, migration, and invasion while promoting apoptosis}

To examine the effects of GOLPH3 on EC cell proliferation, migration, and invasion, the normally invasive KLE EC cell line was transfected with shGOLPH3 or empty vector. KLE cells transfected with shGOLPH3 plasmid displayed significantly lower GOLPH3 expression at the mRNA and protein levels compared to control cells transfected with empty vector (Fig. 2A and B), thus confirming transfection efficiency. In addition, cells transfected with shGOLPH3 demonstrated a slower proliferation rate than cells transfected with the empty vector as assessed by the CCK-8 cell viability assay (Fig. 2C). The migration and invasion capacities of KLE cells transfected with shGOLPH3 were also significantly reduced compared to KLE cells transfected with empty vector as determined by transwell migration assays $(p<$ 0.05) (Fig. 2D). To investigate the effect of GOLPH3 
A

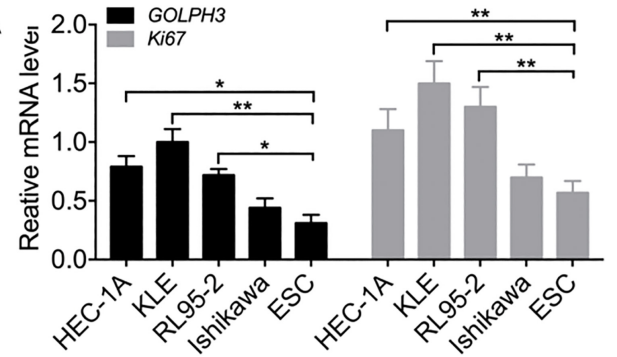

B

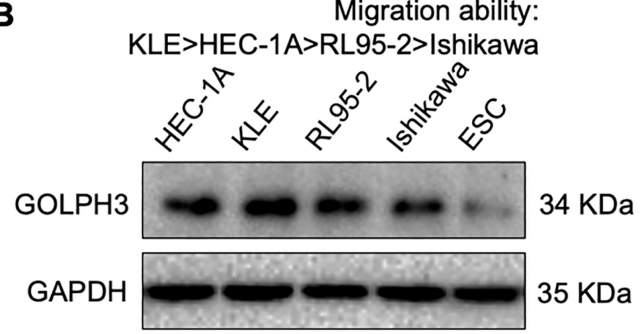

C

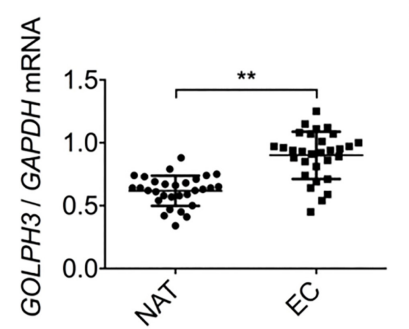

D

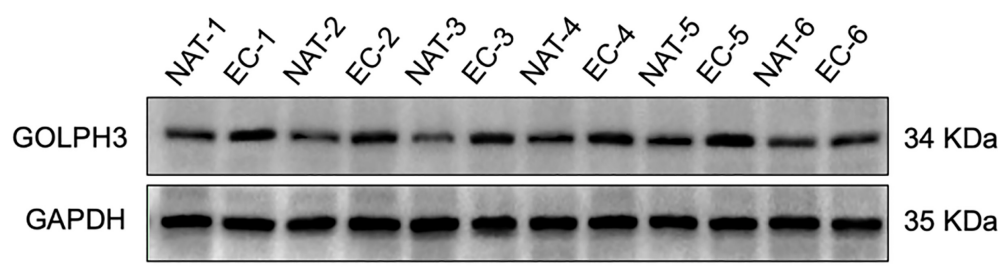

$\mathbf{E}$
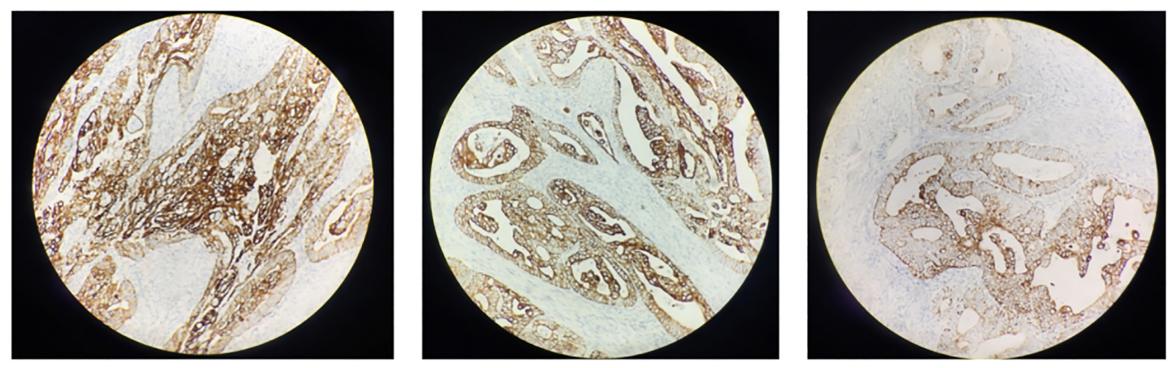

Fig. 1. Expression of GOLPH3 is upregulated in endometrial carcinoma (EC). (A) The mRNA level of GOLPH3 and Ki67 in four EC cell lines (HEC-1A, KLE, RL95-2, and Ishikawa) and endometrial stromal cells (ESCs) as measured by qRT-PCR, Ki67 was detected as positive control. (B) The protein level of GOLPH3 in four EC cell lines and ESCs measured by western blot. (C) GOLPH3 mRNA expression in $30 \mathrm{EC}$ tissue samples and normal adjacent tissue (NAT) analyzed by qRT-PCR. (D) GOLPH3 protein expression in six EC tissues and NAT analyzed by western blot. (E) The expression and localization of GOLPH3 in EC tissue chip detected by IHC Magnification 100×. All values are the mean \pm SD of three independent experiments. ${ }^{*} P<0.05,{ }^{* *} P<0.01$.

on EC cell survival, apoptosis was measured using an Annexin V FITC apoptosis detection kit. Flow cytometry revealed a significantly greater apoptosis rate among KLE cells transfected with shGOLPH3 (Fig. 2E). These results demonstrate that suppression of GOLPH3 expression can reduce EC cell proliferation, migration, and invasion while accelerating apoptosis in vitro.

\subsection{Ectopic overexpression of GOLPH3 accelerates $E C$ cell proliferation, migration, and invasion while inhibiting apoptosis}

We transiently transfected pcDNA3.1 (+)-GOLPH3 plasmid into the normally non-invasive Ishikawa cell line, and confirmed transfection efficiency by qRTPCR and western blot. Compared to Ishikawa cells transfected with empty vector, cells transfected with the pcDNA3.1 (+)-GOLPH3 plasmid displayed significantly greater GOLPH3 expression at both mRNA and protein levels (Fig. 3A and B) as well as accelerated proliferation, migration, and invasion $(p<$ 0.05) (Fig. 3C and D). Conversely, Ishikawa cells overexpressing GOLPH3 exhibited a lower apoptosis rate than controls (Fig. 3E). These results demonstrate that ectopic overexpression of GOLPH3 promotes cell proliferation, migration, and invasion while inhibiting apoptosis of EC cells in vitro.

\subsection{Suppression of GOLPH3 expression reduces EC cell distant metastasis in vivo}

We then investigated the functional relevance of GOLPH3 for metastasis in vivo. A suspension of KLE- 

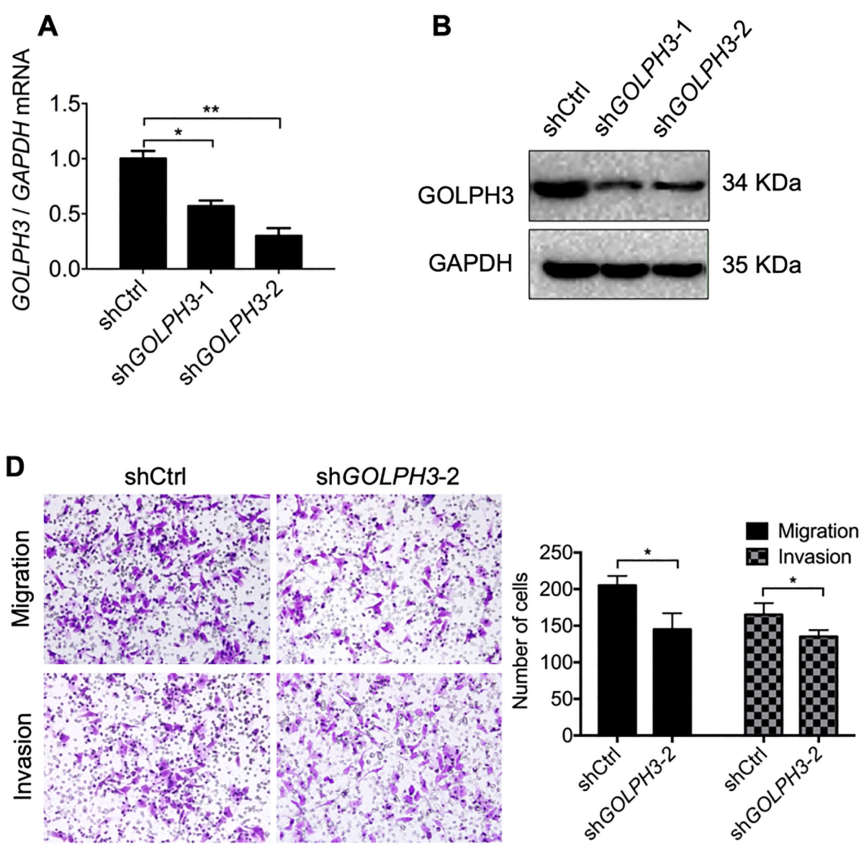

$\mathbf{E}$
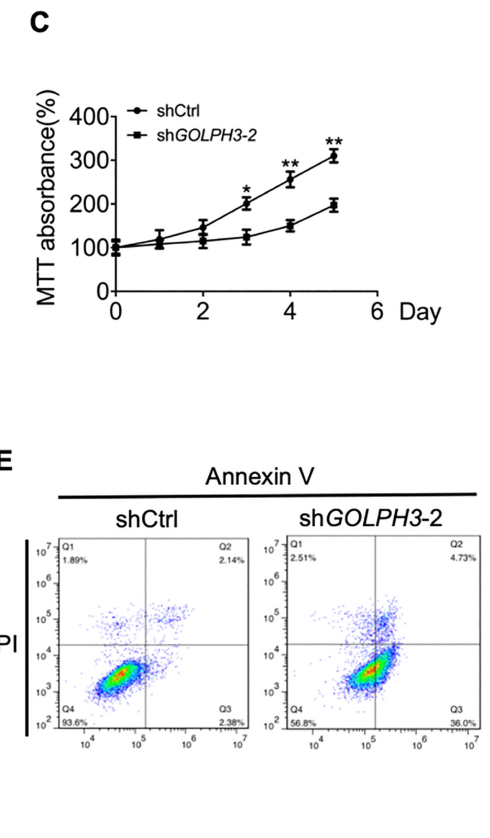

Fig. 2. Downregulation of GOLPH3 in KLE cells reduces cell proliferation, migration, and invasion, and promotes apoptosis in vitro. (A-B) Knockdown of GOLPH3 in KLE cells transfected with shGOLPH3 (KLE-sdGOLPH3 cells) compared to control cells transfected with empty vector was verified by qRT-PCR and western blotting. (C) Serial CCK-8 cell viability assays showing slower proliferation of knockdown cells compared to controls. (D) Downregulation of GOLPH3 also reduced cell migration and invasion in transwell assays. Magnification 100×. (E) Downregulation of GOLPH3 promoted apoptosis of KLE cells as measured by Annexin/PI staining and flow cytometry. All values are the mean \pm SD of three independent experiments. ${ }^{*} P<0.05,{ }^{* *} P<0.01$.

A

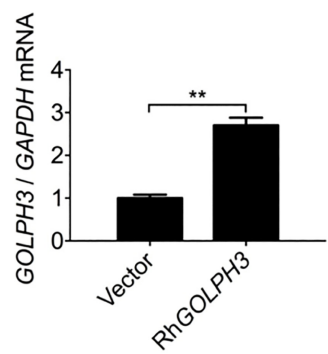

D

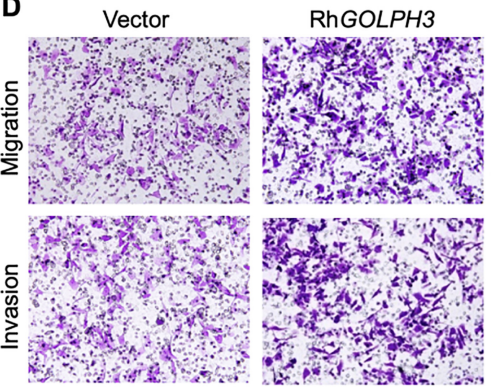

B

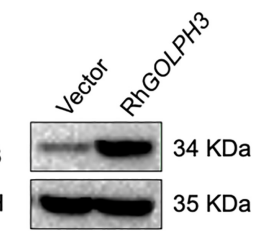

C

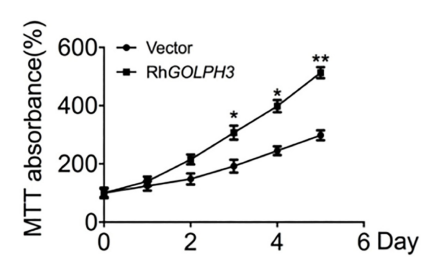

E
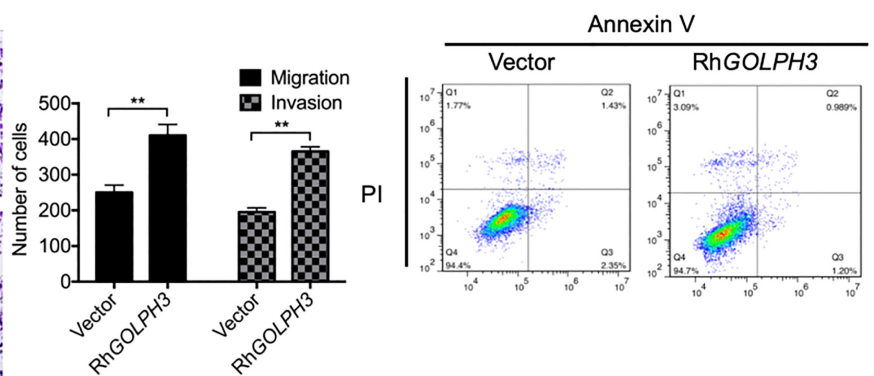

Fig. 3. Ectopic overexpression of GOLPH3 in Ishikawa cells accelerates proliferation, migration, and invasion while inhibiting apoptosis in vitro. (A-B) GOLPH3 overexpression in Ishikawa-RhGOLPH3 cells compared to control cells was verified by qRT-PCR and western blotting. (C) Serial CCK-8 cell viability assays demonstrating faster proliferation rate of Ishikawa-RhGOLPH3 cells versus controls. (D) Overexpression of GOLPH3 also accelerated cell migration and invasion in transwell assays. Magnification 100×. (E) Overexpression of GOLPH3 inhibited apoptosis of Ishikawa cells. All values are the mean $\pm \mathrm{SD}$ of three independent experiments. ${ }^{*} P<0.05,{ }^{* *} P<0.01$. 
A

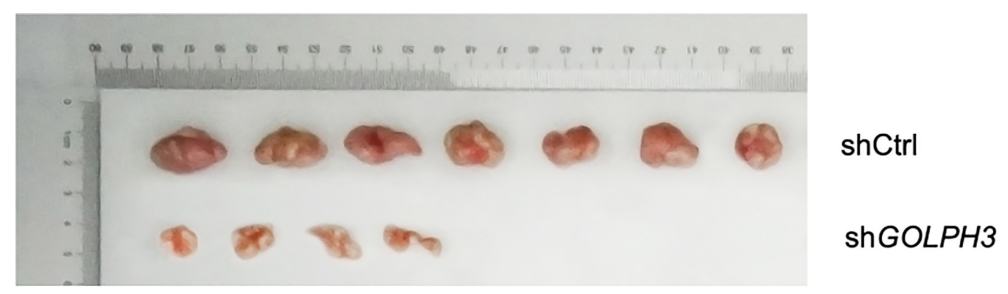

B

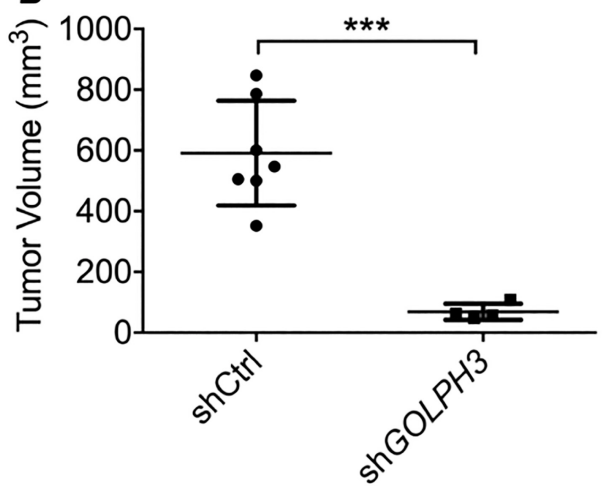

C

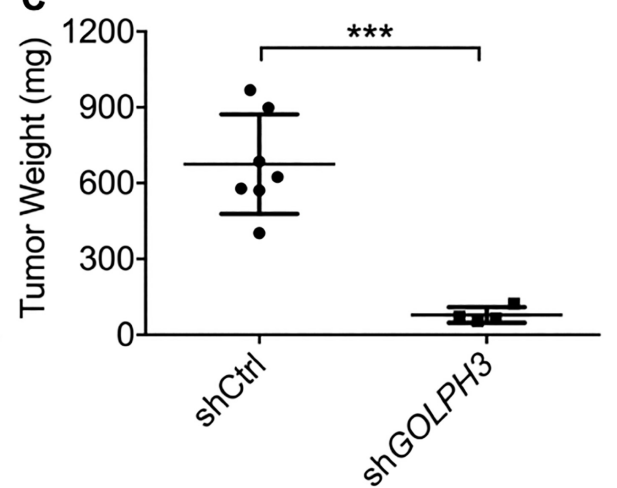

Fig. 4. Downregulation of GOLPH3 reduces distant EC cell metastasis in vivo. The tumor images (A), tumor volume growth curves (B) and tumor weights (C) 30 days after inoculation of nude mice with GOLPH3 knockdown (KLE-shGOLPH3) EC cells or control cells. All values are the mean $\pm \mathrm{SD}$ of three independent experiments. ${ }^{*} P<0.05,{ }^{* *} P<0.01$.

shGOLPH3 (knockdown) or control KLE cells was injected into nude mice through the tail vein, and animals were sacrificed 30 days later. Knockdown of GOLPH3 significantly decreased the number of mice with distant metastases (4 of 7) compared to the control group (7/7) (Fig. 4A). In addition, tumor volume and tumor weight were significantly lower in the KLEshGOLPH3 injection group (Fig. 4B and C). Therefore, elevated GOLPH3 expression appears essential for EC cell metastasis in vivo.

\subsection{GOLPH3 induces epithelial-mesenchymal transition in EC cells}

The EMT is critical for cancer cell migration and invasion [24]. To further support the proposal that GOLPH3 facilitates migration and invasion of EC cells, we examined the mRNA and protein levels of EMT-related genes in overexpressing and knockdown EC cells by qRT-PCR and western blotting. As shown in Fig. 5A and B, compared to the control group, knockdown of GOLPH3 in KLE cells inhibited mRNA and protein expression of the mesenchymal markers $\mathrm{N}$-cadherin and vimentin, and stimulated the expression of the epithelial markers E-cadherin and $\alpha$ catenin. Also consistent with regulation of invasiveness via EMT, ectopic overexpression of GOLPH3 in
Ishikawa cells increased expression of mesenchymal markers and decreased expression of epithelial markers (Fig. 5C and D).

\section{Discussion}

Endometrial carcinoma (EC) is the most common malignancy of the female reproductive tract in developed countries and incidence continues to increase [1]. Recent studies indicate that early frequent metastasis that occurs in about one fourth of the high-risk of ECs and is the major reason for poor outcome [25,26]. Therefore, identifying novel molecules that regulate EC metastasis will aid in the identification of molecular prognostic factors and the development of more efficacious treatment strategies. There is a large body of evidence that induction of EMT confers mobility and invasive capacity to epithelial cancer cells, including EC cells, thereby facilitating the generation of distant metastases [27].

In this study, we show for the first time that GOLPH3 is overexpressed in EC tissues and EC cells compared to adjacent non-cancerous tissues, and GOLPH3 was localized in the cytoplasm of carcinoma tissues and the expression level of GOLPH3 was related to the grade of the tumor. In addition, GOLPH3 overexpression is critical for the enhanced proliferative and inva- 
A

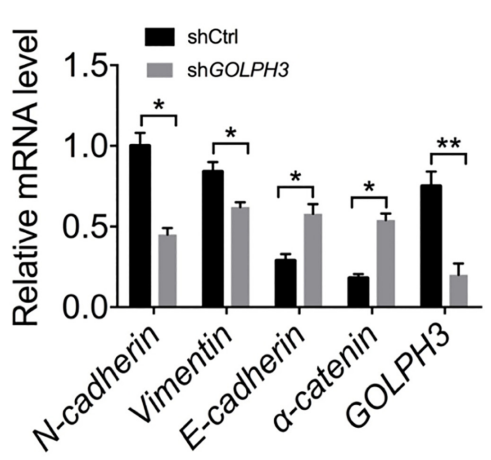

C

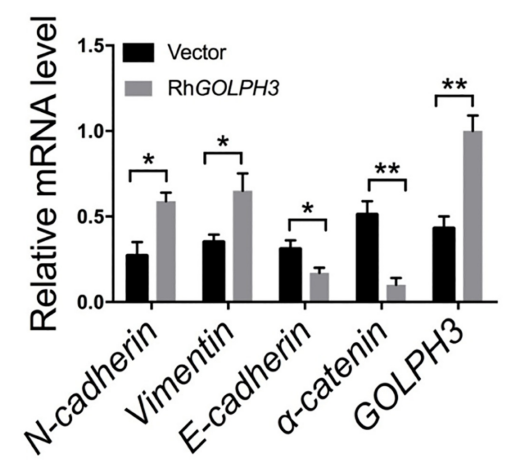

B

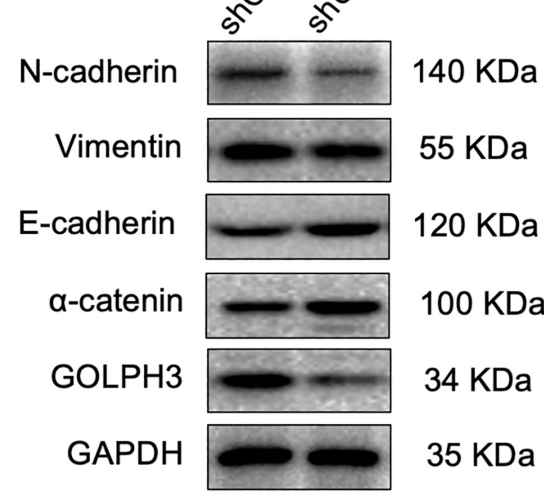

D

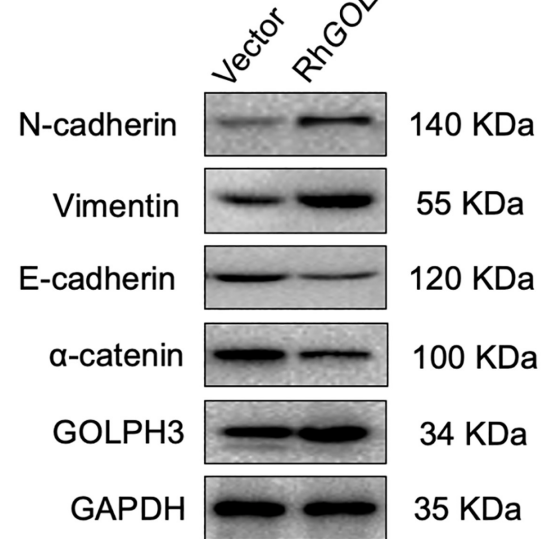

Fig. 5. GOLPH3 regulates the transition between epithelial and mesenchymal phenotypes in EC cells. (A-B) Expression levels of epithelial and mesenchymal markers were compared between KLE-shGOLPH3 (knockdown) and control cells by qRT-PCR and western blotting. GOLPH3 knockdown reduced the expression of mesenchymal markers and upregulated epithelial markers (MET). (C-D) Expression levels of epithelial and mesenchymal marker were compared between overexpressing Ishikawa-RhGOLPH3 and control cells by qRT-PCR and western blotting. Ectopic overexpression of GOLPH3 upregulated mesenchymal marker expression and downregulated epithelial marker expression. All values are the mean $\pm \mathrm{SD}$ of three independent experiments. ${ }^{*} P<0.05,{ }^{* *} P<0.01$.

sive properties as well as reduced apoptosis rate characteristic of cancer cells, in vitro and in animal models. Alternatively, downregulation of GOLPH3 in normally aggressive and invasive EC cells inhibited proliferation and metastatic capacity in vitro and in vivo. We also confirmed that GOLPH3 promotes EC cell metastasis by regulating EMT, as EC cells strongly expressing GOLPH3 displayed an EMT phenotype, while downregulation in aggressive EC cells led to mesenchymalepithelial transition (MET). This study therefore provides important evidence that GOLPH3 overexpression is critical for EC cell tumorigenicity, aggressiveness and metastasis capacity. While EMT is essential for EC cell dissemination and seeding of new tu- mors at distant sites [28], our results also demonstrate that these GOLPH3-induced processes are reversible by suppression of GOLPH3 expression. Therefore, GOLPH3 may serve as a novel diagnostic and therapeutic target, strongly warranting further investigation.

Previous studies reported that GOLPH3 enhances proliferation and metastasis of various cancer cells by activating the targets of $\mathrm{Wnt} / \beta$-catenin, mTOR, and NF-kB signaling pathways [17-19]. All these studies support our findings regarding the oncogenic functions of GOLPH3 in EC cells, although the intervening signaling pathways and downstream targets remain to be identified. 
In conclusion, this study demonstrates that relative overexpression of GOLPH3 in EC cells confers an aggressive phenotype characterized by rapid proliferation, strong invasive capacity, and low apoptosis rate. More importantly, these features may be reversed by suppression of GOLPH3 expression, underscoring the potential therapeutic value of strategies targeting GOLPH3 signaling. But unfortunately, we haven't measured the bioluminescence image of xenografted tumors and intensity, and these results should be confirmed with further clinical studies on human patients.

\section{Conflict of interest}

The authors have no conflicts of interest to declare.

\section{References}

[1] P. Morice et al., Endometrial cancer, The Lancet 387 (2016), 1094-1108.

[2] K.J. Dedes et al., Emerging therapeutic targets in endometrial cancer, Nature Reviews Clinical Oncology 8 (2011), 261.

[3] D. Thanapprapasr and K. Thanapprapasr, Molecular therapy as a future strategy in endometrial cancer, Asian Pacific Journal of Cancer Prevention 14 (2013), 3419-3423.

[4] Y.C. Lee, S. Lheureux and A.M. Oza, Treatment strategies for endometrial cancer: current practice and perspective, Current Opinion in Obstetrics and Gynecology 29 (2017), 47-58.

[5] H.C. Dippold et al., GOLPH3 Bridges Phosphatidylinositol4-Phosphate and Actomyosin to Stretch and Shape the Golgi to Promote Budding, Cell 139 (2009), 337-351.

[6] C.C. Wu et al., GMx33: a novel family of trans-Golgi proteins identified by proteomics, Traffic 1 (2000), 963-975.

[7] S. Tang et al., GOLPH3: a novel biomarker that correlates with poor survival and resistance to chemotherapy in breast cancer, Oncotarget 8 (2017), 105155-105169.

[8] W. Tang et al., Overexpression of GOLPH3 is associated with poor survival in Non-small-cell lung cancer, American Journal of Translational Research 8 (2016), 1756.

[9] Z. Zeng et al., Overexpression of GOLPH3 promotes proliferation and tumorigenicity in breast cancer via suppression of the FOXO1 transcription factor, Clinical Cancer Research $\mathbf{1 8}$ (2012), 4059-4069.

[10] J. Sun et al., GOLPH3 induces epithelial-mesenchymal transition via $\mathrm{Wnt} / \beta$-catenin signaling pathway in epithelial ovarian cancer, Cancer Medicine 6 (2017), 834-844.
[11] H. Li et al., GOLPH3 overexpression correlates with tumor progression and poor prognosis in patients with clinically N0 oral tongue cancer, Journal of Translational Medicine $\mathbf{1 0}$ (2012), 168

[12] Q. Li, Y. Ma and W. Xu, High GOLPH3 expression is associated with poor prognosis and invasion of hepatocellular carcinoma, Molecular Medicine Reports 11 (2015), 4315-4320.

[13] K. Zhu et al., GOLPH3 overexpression correlates with poor response to neoadjuvant therapy and prognosis in locally advanced rectal cancer, Oncotarget 7(2016), 68328.

[14] X. Hua et al., Increased expression of Golgi phosphoprotein-3 is associated with tumor aggressiveness and poor prognosis of prostate cancer, Diagnostic Pathology 7 (2012), 127.

[15] Q. Zhang et al., GOLPH3 is a potential therapeutic target and a prognostic indicatior of poor survival in bladder cancer treated by cystectomy, Oncotarget 6 (2015), 32177-32192.

[16] J.H. Wang et al., High Expression of GOLPH3 in Esophageal Squamous Cell Carcinoma Correlates with Poor Prognosis, PLOS ONE 7 (2012), e45622.

[17] Y. Liu, Y. Sun and A. Zhao, MicroRNA-134 suppresses cell proliferation in gastric cancer cells via targeting of GOLPH3, Oncology Reports 37 (2017), 2441-2448.

[18] J. Peng et al., Mechanisms of GOLPH3 Associated with the Progression of Gastric Cancer: A Preliminary Study, PLOS ONE 9 (2014), e107362.

[19] Y. Xue et al., GOLPH3 is a novel marker of poor prognosis and a potential therapeutic target in human renal cell carcinoma, British Journal of Cancer 110 (2014), 2250-2260.

[20] K.L. Scott et al., GOLPH3 modulates mTOR signalling and rapamycin sensitivity in cancer, Nature 459 (2009), 10851090

[21] T. Dai et al., Golgi phosphoprotein 3 (GOLPH3) promotes hepatocellular carcinoma cell aggressiveness by activating the NF- $\kappa$ B pathway, Journal of Pathology 235 (2015), 490-501.

[22] C.Z. Qiu et al., Correlation of GOLPH3 Gene with Wnt Signaling Pathway in Human Colon Cancer Cells, Journal of Cancer 7 (2016), 928-934.

[23] Z. Hou et al., Role of interleukin-1 receptor type II in the pathogenesis of endometriosis, Fertility \& Sterility 89 (2008), 42-51.

[24] S. Heerboth et al., EMT and tumor metastasis, Clinical \& Translational Medicine 4(2015), 6.

[25] N. Colombo et al., ESMO-ESGO-ESTRO Consensus Conference on Endometrial Cancer, Ann Oncol 27 (2016), 16-41.

[26] J.P. Thiery et al., Epithelial-mesenchymal transitions in development and disease, Cell 139 (2009), 871-890.

[27] S. Floor et al., Cancer cells in epithelial-to-mesenchymal transition and tumor-propagating-cancer stem cells: Distinct, overlapping or same populations, Oncogene 30 (2011), 46094621.

[28] A. Makker and M.M. Goel, Tumor progression, metastasis, and modulators of epithelial-mesenchymal transition in endometrioid endometrial carcinoma: an update, Endocr Relat Cancer 23 (2016), R85-R111. 